\title{
Characterization and functional analysis of seven flagellin genes in Rhizobium leguminosarum bv. viciae. Characterization of $R$. leguminosarum flagellins
}

\author{
Dinah D Tambalo ${ }^{1}$, Denise E Bustard ${ }^{1}$, Kate L Del Bel', Susan F Koval ${ }^{2}$, Morgan F Khan ${ }^{3}$, Michael F Hynes ${ }^{1 *}$
}

\begin{abstract}
Background: Rhizobium leguminosarum bv. viciae establishes symbiotic nitrogen fixing partnerships with plant species belonging to the Tribe Vicieae, which includes the genera Vicia, Lathyrus, Pisum and Lens. Motility and chemotaxis are important in the ecology of $R$. leguminosarum to provide a competitive advantage during the early steps of nodulation, but the mechanisms of motility and flagellar assembly remain poorly studied. This paper addresses the role of the seven flagellin genes in producing a functional flagellum.

Results: $R$. leguminosarum strains 3841 and VF39SM have seven flagellin genes (flaA, flaB, flaC, flaD, flaE, flaH, and flaG), which are transcribed separately. The predicted flagellins of 3841 are highly similar or identical to the corresponding flagellins in VF39SM. flaA, flaB, flaC, and flaD are in tandem array and are located in the main flagellar gene cluster. flaH and flaG are located outside of the flagellar/motility region while flaE is plasmid-borne. Five flagellin subunits (FlaA, FlaB, FlaC, FlaE, and $\mathrm{FlaG}$ ) are highly similar to each other, whereas $\mathrm{FlaD}$ and $\mathrm{FlaH}$ are more distantly related. All flagellins exhibit conserved amino acid residues at the $\mathrm{N}$ - and $\mathrm{C}$-terminal ends and are variable in the central regions. Strain 3841 has 1-3 plain subpolar flagella while strain VF39SM exhibits 4-7 plain peritrichous flagella. Three flagellins (FlaA/B/C) and five flagellins (FlaA/B/C/E/G) were detected by mass spectrometry in the flagellar filaments of strains 3841 and VF39SM, respectively. Mutation of flaA resulted in nonmotile VF39SM and extremely reduced motility in 3841. Individual mutations of flaB and flaC resulted in shorter flagellar filaments and consequently reduced swimming and swarming motility for both strains. Mutant VF39SM strains carrying individual mutations in flaD, flaE, flaH, and flaG were not significantly affected in motility and filament morphology. The flagellar filament and the motility of 3841 strains with mutations in flaD and flaG were not significantly affected while flaE and flaH mutants exhibited shortened filaments and reduced swimming motility.
\end{abstract}

Conclusion: The results obtained from this study demonstrate that FlaA, FlaB, and $\mathrm{FlaC}$ are major components of the flagellar filament while FlaD and FlaG are minor components for R. leguminosarum strains 3841 and VF39SM. We also observed differences between the two strains, wherein FlaE and FlaH appear to be minor components of the flagellar filaments in VF39SM but these flagellin subunits may play more important roles in 3841 . This paper also demonstrates that the flagellins of 3841 and VF39SM are possibly glycosylated.

\footnotetext{
* Correspondence: hynes@ucalgary.ca

'Department of Biological Sciences, University of Calgary. 2500 University

Drive NW. Calgary, AB, T2N 1N4, Canada

Full list of author information is available at the end of the article
}

C 2010 Tambalo et al; licensee BioMed Central Ltd. This is an Open Access article distributed under the terms of the Creative 


\section{Background}

Motility is an important property of bacteria that enables them to move towards favorable growth conditions and away from detrimental conditions. Most bacteria move through the use of flagella. A bacterial flagellum consists of three distinct regions: the basal body, the hook, and the filament [1]. Flagellar assembly and motility are well-understood in enteric bacteria, particularly Escherichia coli and Salmonella. The flagellar filament of $E$. coli is a helical arrangement of as many as 20,000 flagellin subunits, whose molecular weight is approximately $50 \mathrm{kDa}[1,2]$. Whereas the $E$. coli flagellar filament consists of one type of flagellin $[3,4]$, the presence of more than one flagellin type has been reported for a few soil bacteria, including Sinorhizobium meliloti, Rhizobium lupini, and Agrobacterium tumefaciens [5-10]. S. meliloti and A. tumefaciens assemble their flagellar filaments from four closely related flagellin subunits (FlaA, FlaB, FlaC, and FlaD) while $R$. lupini flagella consist of three flagellin subunits (FlaA, FlaB, and FlaD). For these soil bacteria, FlaA is the principal flagellin subunit of the flagellar filament while the other subunits play minor roles.

The flagellar filament is a highly conserved structure in terms of amino acid composition, subunit domain organization of the flagellin monomers, and the symmetry and mode of assembly $[11,12]$. The quaternary structure of the flagellar filament has been divided into four structural domains, domain 0 (D0) to domain 3 (D3), and the amino acid residues of the flagellin protein have been assigned to these domains [13-17]. Domains D0 and D1, which are found in the filament core, correspond to the amino and carboxy terminal residues. Domains D2 and D3, the outer region of the filament, consist of the flagellin central residues. The amino acid sequences corresponding to domains 0 and 1 are highly conserved across different bacterial strains [14,18], and were shown to be essential in the polymerization of bacterial flagellar filaments [19]. Domains D2 and D3, on the other hand are considerably variable in amino acid composition and are generally not well-aligned [18]. Domain D3 of the filament contributes to filament stability [16] but it can be deleted or reduced in size without severely impairing filament assembly and function [16,20-22].

Flagellar filaments are traditionally classified as either "plain" or "complex". Plain filaments are often found in enterobacteria, such as Salmonella typhimurium and E. coli $[23,24]$. These filaments have a smooth surface and are able to change from left- to right-handedness or from a counterclockwise to a clockwise direction of rotation [5]. A few soil bacteria such as Pseudomonas rhodos [25], R. lupini [24,26] and S. meliloti [26] are equipped with one or more complex flagella. Studies have shown that transmission electron microscopy can be used to differentiate between plain and complex flagella $[24,27]$. Complex flagellar filaments have a distinct ridging pattern while plain filaments appear thinner and have little to no visible external pattern. The complex filaments are also more rigid and more brittle than the plain filament. It is thought that increased rigidity is favorable for motility in viscous environment such as in the soil biotope [27].

To date, little is known about the flagellar filament of Rhizobium leguminosarum bv. viciae. A previous study has shown that the movement of $R$. leguminosarum bv. viciae strain 3841 is propelled by one or two subpolar flagella [28]. The same study has also suggested that the flagella rotate in a unidirectional pattern and the direction of movement is changed by modulating the rotary speed. In this paper, we characterize the genes encoding the seven flagellin subunits in $R$. leguminosarum bv. viciae. We have conducted sequence analysis, as well as mutational and transcriptional studies to determine the roles of the flagellin genes in flagellar assembly and function for the sequenced strain 3841 and our laboratory strain VF39SM. We have studied the flagellin genes in parallel in both strains because the two strains exhibit differences in pattern of flagellation (see below) and also in swarming motility (below and [29]).

\section{Methods}

\section{Bacterial strains, plasmids, and growth conditions}

The bacterial strains and plasmids used in this study are shown in Table 1. R. leguminosarum and E. coli strains were grown in TY medium [30] and LB medium [31], respectively. The concentrations of antibiotics used to grow $R$. leguminosarum were streptomycin $(\mathrm{Sm}) 500 \mu \mathrm{g} /$ $\mathrm{ml}$, gentamicin $(\mathrm{Gm}) 30 \mu \mathrm{g} / \mathrm{ml}$, neomycin $(\mathrm{Nm}) 100 \mu \mathrm{g} /$ $\mathrm{ml}$, spectinomycin (Sp) $100 \mu \mathrm{g} / \mathrm{ml}$, and tetracycline (Tc) $5 \mu \mathrm{g} / \mathrm{ml}$. E. coli strains were grown in the following antibiotic concentrations: ampicillin (Ap) $100 \mu \mathrm{g} / \mathrm{ml}$, kanamycin $(\mathrm{Km}) 50 \mu \mathrm{g} / \mathrm{ml}$, gentamicin $(\mathrm{Gm}) 15 \mu \mathrm{g} / \mathrm{ml}$, and tetracycline (Tc) $10 \mu \mathrm{g} / \mathrm{ml}$.

\section{Recombinant DNA techniques}

Recombinant DNA techniques were performed using standard methods [31]. Restriction endonucleases used in this study were purchased from Invitrogen or New England Biolabs and used according to the manufacturer's specifications. DNA fragments were isolated from agarose gels using Qiaquick Gel Extraction kit (Qiagen). Plasmids were isolated from E. coli strains using GeneJET $^{\mathrm{TM}}$ Plasmid Miniprep kit (Fermentas Life Sciences). Total DNA was isolated from $R$. leguminosarum strains using Aquapure Genomic DNA Isolation kit (Bio-Rad 
Table 1 Bacterial strains and plasmids used in the study

\begin{tabular}{|c|c|c|}
\hline Strains and Plasmids & Relevant characteristics & $\begin{array}{l}\text { Source or } \\
\text { Reference }\end{array}$ \\
\hline \multicolumn{3}{|l|}{ Escherichia coli strains } \\
\hline $\mathrm{DH} 5 \alpha$ & endA1, hsdR17, supE44, thi-1, recA1, gyrA96, relA1,(argF-lacZYA), U169, $\phi$ 80dlacZ $\triangle \mathrm{M} 15$ & Invitrogen \\
\hline S17.1 & Spr. RP4 tra region, mobilizer strain & [69] \\
\hline \multicolumn{3}{|l|}{$\begin{array}{l}\text { Rhizobium leguminosarum } \\
\text { strains }\end{array}$} \\
\hline 3841 & biovar viciae, JB300 derivative, $\mathrm{Sm}^{r}$ & {$[70]$} \\
\hline VF39SM & biovar viciae, $\mathrm{Sm}^{r}$ & {$[71]$} \\
\hline VF39SM flaA & VF39SM flaA', Sm ${ }^{r}, \mathrm{Nm}^{r}$ & This work \\
\hline VF39SMflaA ${ }^{+}$ & VF39SMflaA complemented with flaA, $\mathrm{Sm}^{r}, \mathrm{Nm}^{r}, \mathrm{Gm}^{r}$ & This work \\
\hline $3841 \mathrm{flaA}^{-}$ & gusA-Nm cassette insertion in $3841 \mathrm{flaA}, \mathrm{Sm}^{r}, \mathrm{Nm}^{r}$ & This work \\
\hline $3841 f l a A^{+}$ & 3841 flaA complemented with flaA, $\mathrm{Sm}^{r}, \mathrm{Nm}^{r}, \mathrm{Gm}^{r}$ & This work \\
\hline VF39SM flaB & Spectinomycin cassette insertion in VF39SM flaB, Sm ${ }^{r}, \mathrm{Sp}^{r}$ & This work \\
\hline $3841 \mathrm{flaB}^{-}$ & Spectinomycin cassette insertion in $3841 \mathrm{flaB}, \mathrm{Sm}^{r}, \mathrm{Sp}^{r}$ & This work \\
\hline VF39SM flaC & gusA-Nm cassette insertion in VF39SM flaC, $\mathrm{Sm}^{r}, \mathrm{Nm}^{r}$ & This work \\
\hline $3841 \mathrm{flaC}$ & gusA-Nm cassette insertion in $3841 \mathrm{flaC}, \mathrm{Sm}^{r}, \mathrm{Nm}^{r}$ & This work \\
\hline VF39SM flaD- & gusA-Nm cassette insertion in VF39SM flaD, $\mathrm{Sm}^{r}, \mathrm{Nm}^{r}$ & This work \\
\hline $3841 \mathrm{flaD}^{-}$ & gusA-Nm cassette insertion in $3841 \mathrm{flaD}, \mathrm{Sm}^{r}, \mathrm{Nm}^{r}$ & This work \\
\hline VF39SM flaE & gusA-Nm cassette insertion in VF39SM flaE, $\mathrm{Sm}^{r}, \mathrm{Nm}^{r}$ & This work \\
\hline 3841 flaE & gusA-Nm cassette insertion in $3841 \mathrm{flaE}, \mathrm{Sm}^{r}, \mathrm{Nm}^{r}$ & This work \\
\hline VF39SM flaH & Neomycin-resistance cassette insertion in VF39SM flaH, $\mathrm{Sm}^{r}, \mathrm{Nm}^{r}$ & This work \\
\hline $3841 \mathrm{flaH}^{-}$ & Neomycin-resistance cassette insertion in $3841 \mathrm{flaH}, \mathrm{Sm}^{r}, \mathrm{Nm}^{r}$ & This work \\
\hline VF39SM flaG & Tetracycline-resistance cassette insertion in VF39SM flaG, $\mathrm{Sm}^{r}, T c^{r}$ & This work \\
\hline $3841 \mathrm{flaG}$ & Tetracycline-resistance cassette insertion in $3841 \mathrm{flaG}, \mathrm{Sm}^{r}, \mathrm{Tc}^{r}$ & This work \\
\hline $3841 \mathrm{flaA} / \mathrm{B} / \mathrm{C} / \mathrm{D}^{-}$ & 3841 strain with mutations in flaAvB/C/D, Sm ${ }^{r}, \mathrm{Nm}^{r}$ & This work \\
\hline VF39SM flaA/B/C/D- & VF39SM strain with mutations in flaB/C/D, Sm ${ }^{r}, \mathrm{Nm}^{r}$ & This work \\
\hline VF39SM flaB/C/D- & VF39SM flaA/B/C/D - complemented with flaA; $\mathrm{Sm}^{r}, \mathrm{Nm}^{r}, \mathrm{Gm}^{r}$ & This work \\
\hline $3841 \mathrm{flaB} / \mathrm{C} / \mathrm{D}^{-}$ & 3841 flaA/B/C/D - complemented with flaA; $\mathrm{Sm}^{r}, \mathrm{Nm}^{r}, \mathrm{Gm}^{r}$ & This work \\
\hline \multicolumn{3}{|l|}{ Plasmids } \\
\hline pCR2.1-TOPO & Cloning vector, $\mathrm{Amp}^{r}, \mathrm{Km}^{r}$ & Invitrogen \\
\hline pJQ200SK & Suicide vector with $s a c B$ system; $G m^{r}$ & {$[32]$} \\
\hline pJQ200mp18 & Suicide vector with sacB system; $G m^{r}$ & [32] \\
\hline pCRS530 & Contains a promoterless gusA-Nm cassette & [33] \\
\hline pBSL99 & Contains kanamycin-resistance cassette & [36] \\
\hline pBSIISK+ & Cloning vector, $A m p^{r}$ & Stratagene \\
\hline pBS::flaD3'-Km-flaA5' & flaA5' fragment (from pCR2.1:flaA5') subcloned into pBS::flaD3'-Km, $\mathrm{Amp}^{r}, \mathrm{Km}^{r}$ & This work \\
\hline pJQmp18:: flaD3'-Km-flaA5' & flaD3'-Km-flaA5' fragment subcloned from pBS::flaD3'-Km-flaA5' into pJQmp18, $\mathrm{Gm}^{r}, \mathrm{Km}^{r}$ & This work \\
\hline $\mathrm{pHP} 45 \Omega$ & Contains omega-spectinomycin cassette; $S p^{r}$ & [34] \\
\hline $\mathrm{pHP} 45: \Omega T \mathrm{C}$ & Contains tetracycline-resistance cassette; $T c^{r}$ & [35] \\
\hline pBBR1-MCS5 & Broad-host-range cloning vector, $\mathrm{Gm}^{r}$ & [72] \\
\hline pFus1 & pMP220 derivative with promoterless gus $A, T C^{r}$ & [33] \\
\hline pBBR1-MCS5:3841flaA & Broad-host-range cloning vector containing flaA gene (with promoter region) from 3841 & This work \\
\hline pBBR1-MCS5:VF39SMflaA & $\begin{array}{l}\text { Broad-host-range cloning vector containing flaA gene (with promoter region) from } \\
\text { VF39SM }\end{array}$ & This work \\
\hline pFus1::flaB & flaB promoter introduced into pFus1, $T c^{r}$ & This work \\
\hline
\end{tabular}


Laboratories). Primers were synthesized by Sigma Genosys (Sigma-Aldrich) and amplification was carried out using a Multi GeneII PCR machine (Labnet International, Inc.). Southern blots were performed using a non-radioactive technique with reagents and protocols supplied by Roche Applied Science.

\section{Mutagenesis of flagellin genes}

The seven fla genes were PCR amplified from $R$. leguminosarum using the primers listed in Additional file 1. The PCR products were individually cloned into the vector pCR2.1-TOPO using the TOPO Cloning kit (Invitrogen). The genes were excised from the TOPO vector and then ligated into either PJQ200SK or pJQ200mp18 [32]. The details on constructing the individual fla mutants are presented in Additional file 2 . Individual mutations in flaA, flaC, flaD, and flaE were introduced by inserting a gusA-Nm ${ }^{r}$ (CAS-GNm) cassette from pCRS530 [33] into the reading frame of each gene. The flaB and flaG genes were mutated by inserting a spectinomycin and tetracycline resistance cassette,

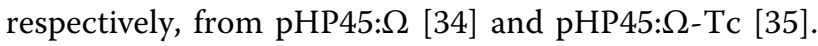
The $f l a H$ gene was mutated by inserting a kanamycinresistance cassette from pBSL99 [36]. The $\mathrm{flaA} / \mathrm{B} / \mathrm{C} / \mathrm{D}$ genes were mutated by separately amplifying the $5^{\prime}$ end of flaA plus flanking region (missing the 3' end of flaA) and the 3 ' end of flaD plus flanking region (missing the 5 ' end of $f l a D$ ). The truncated genes were cloned separately into pCR2.1-TOPO and the resulting plasmids (pCR2.1::flaA5' and pBS::flaD3') were sequenced at the University of Calgary Core DNA Services. The fragment containing the truncated flaD gene was subcloned into pBSIISK+ (Stratagene) creating pBS::flaD3'. A kanamycin-resistance cassette $(\mathrm{Km})$ from pBSL99 [36] was ligated upstream of the flaD3' fragment resulting in the construct $\mathrm{pBS}:$ :flaD3'-Km. The fragment containing the truncated flaA gene (from pCR2.1::flaA5') was subcloned into $\mathrm{pBS}:$ : flaD3'-Km, upstream of the $\mathrm{Km}$-cassette creating $\mathrm{pBS}$ ::flaD3'-Km-flaA5'. A fragment containing the truncated flaA gene, kanamycin resistance cassette, and truncated flaD gene was subcloned from pBS::flaD3'Km-flaA5' into pJQ200mp18 [32] creating PJQmp18:: flaD3'-Km-flaA5'. Each of the mutated gene/s was introduced into the genome of $R$. leguminosarum by homologous recombination. The $f l a A / B / C / D$ mutants have deletions in the following: flaA 3' end; flaB; flaC; and flaD 5' end. Southern hybridization and/or PCR were performed for each gene to confirm replacement of the wild-type gene with the mutated gene/s.

\section{Construction of gene fusions and B-glucuronidase (gusA) reporter gene assays}

The promoter region of $f l a B$ was cloned upstream of a promoterless gusA gene in pFus1 [33]. The resulting construct was introduced into VF39SM and 3841 by biparental mating. VF39SM and 3841 strains containing the flaB-gusA fusion were grown in TY broth for 48 hours at $30^{\circ} \mathrm{C}$ [33]. $\beta$-glucuronidase activity was measured as described by Jefferson et al. [37] and modified by Yost et al. [38]. The data given are the means of triplicate experiments.

\section{Swimming motility test}

The strains were grown in TY broth for 24 hours. Swimming motility was determined by inoculating the strains into a motility medium (YES) containing the following: $0.3 \%$ agar, $0.01 \%$ yeast extract, and $1 \mathrm{mM}$ $\mathrm{MgSO}_{4}$ [39]. The optical densities (OD600) of the cultures were standardized and equal amounts of inoculum were inoculated into the swimming agar using a finepoint pipette tip. The swimming diameter was measured 3-4 days after inoculation.

\section{Swarming Motility Test}

The swarm assay was performed following the method described by Tambalo et al. [29]. Briefly, R. leguminosarum wildtype and fla mutant strains were grown in TY broth for 24 hours. Equal amounts of inoculum from the TY culture was used to inoculate swarm plates. The plates were incubated at $22^{\circ} \mathrm{C}$ for two to three weeks and the swarming motility of the fla mutants was compared with the wildtype.

\section{Flagellar filament isolation}

Flagellin proteins were isolated from $R$. leguminosarum based on the procedure described by Maruyama et al. [40]. Cells were grown in $100 \mathrm{ml}$ of TY broth for 48 hours with slow agitation $(50 \mathrm{rpm})$. The bacterial cells were collected by centrifugation at $12,000 \times \mathrm{g}$ for 10 minutes. The pellet was resuspended in $40 \mathrm{mM}$ phosphate buffer. The bacterial cells were vigorously agitated using a vortex to detach the flagella from the cells. The mixture was centrifuged at $12,000 \times \mathrm{g}$ for 10 minutes using a Sorval centrifuge. The supernatant was removed and centrifuged again at the same speed and time. The supernatant containing the detached flagella was centrifuged in an ultracentrifuge at 50,000 $\times \mathrm{g}$ for 2 hours. The pellet was resuspended in $200 \mu \mathrm{L}$ of $40 \mathrm{mM}$ phosphate buffer.

\section{Immunoblot}

The flagellar protein samples were denatured at $100^{\circ} \mathrm{C}$ for 5 minutes and then separated on $12 \%$ acrylamide SDS-PAGE gel at $200 \mathrm{~V}$ for 45 minutes. Molecular size markers from Bio-Rad and Fermentas were used. After electrophoresis, the gel was blotted onto a PVDF membrane (Bio-Rad) using the Bio-Rad apparatus and protocol for electrophoretic transfer. The blot was blocked 
with $10 \%$ skim milk solution for 2 hours. After washing with phosphate-buffered saline (PBS) solution, the blot was probed overnight using a polyclonal flagellar antibody raised in a rabbit against isolated flagellar filaments [41]. Protein A-alkaline phosphatase (Sigma-Aldrich) was used as the secondary antibody. The blot was washed with PBS and was developed using NBT/BCIP (Sigma).

\section{Preparation of samples for tandem mass spectrometry analysis (MS/MS)}

The flagellar protein samples were run on a polyacrylamide gel as described above. Staining and destaining of the protein gel were performed following standard protocols [42]. The gel was soaked overnight in a staining solution containing 0.1\% Coomassie Brilliant Blue (R250; Sigma), $40 \%$ methanol, and $10 \%$ acetic acid. Destaining was done using a solution containing $40 \%$ methanol and $10 \%$ acetic acid. The bands (between approximately $25-37 \mathrm{kDa}$ ) were excised and submitted to the Southern Alberta Mass Spectrometry (SAMS) Centre at the University of Calgary for LC-MS/MS analysis. Two bands within the size range were observed in the gel. The two bands were analyzed separately for 3841 and in combination for VF39SM.

The gel slices were rinsed once with HPLC-grade water and then twice with $25 \mathrm{mM}$ ammonium bicarbonate in $50 \%(\mathrm{v} / \mathrm{v})$ acetonitrile. The gel slices were dehydrated with acetonitrile prior to lyophilization. The dehydrated gel was resuspended in $25 \mathrm{mM}$ ammonium bicarbonate (pH8.0) and samples were digested with trypsin. The peptides were extracted from the gel using $1 \%$ formic acid in $50 \%$ acetonitrile. The extracts were reduced to dryness and then reconstituted in mobile phase of the buffer ( $3 \%$ acetonitrile with $0.2 \%$ formic acid) for liquid chromatography.

\section{Tandem mass spectrometry analysis (MS/MS)}

The digests were analyzed using an integrated Agilent 1100 LC-Ion-Trap-XCT-Ultra system (Agilent Technologies, Santa Clara, CA), which has an integrated fluidic cartridge for peptide capture, separation, and nanospraying (HPLC Chip). The injected samples were trapped and desalted for 5 minutes using a pre-column channel (40-nl volume; Zorbax $\left.300 \mathrm{SB}-\mathrm{C}_{18}\right)$ with an auxiliary pump that delivers $3 \%$ acetonitrile and $0.2 \%$ formic acid at a flowrate of $4 \mu \mathrm{l} /$ minute. The peptides were reverse-eluted from the trapping column and separated on a $150 \mathrm{~mm}$-long analytical column (Zorbax $\left.300 \mathrm{SB}-\mathrm{C}_{18}\right)$ at a flowrate of $0.3 \mu \mathrm{l} /$ minute. The peptides were eluted using a $5-70 \%(\mathrm{v} / \mathrm{v})$ acetonitrile gradient in $0.2 \%(\mathrm{v} / \mathrm{v})$ formic acid over a period of 10 minutes. The MS/MS spectra were collected by data-dependent acquisition, with parent ion scans of $8100 \mathrm{Th} / \mathrm{s}$ over $\mathrm{m} / \mathrm{z}$
400-2,000. MS/MS scans at the same rate over $\mathrm{m} / z$ 1002200.

\section{Mass Spectrometry Data Analysis}

DataAnalysis software for the 6300 series ion trap, v3.4 (build 175) was used to extract the peak-list data. The MS/MS data were analyzed using Mascot v2.1 (Matrix Science, Boston, MA) with the following parameters: 1.6 Da precursor ion mass tolerance, $0.8 \mathrm{Da}$ fragment ion mass tolerance, and one potential missed cleavage. A protein database for $R$. leguminosarum 3841 was obtained from the Wellcome Trust Sanger Institute website $\mathrm{ftp}: / / \mathrm{ftp}$.sanger.ac.uk/pub/pathogens/rl/ and was deposited in Mascot. The deposited $R$. leguminosarum 3841 protein database was used for database searching to identify the proteins present in the flagellar preparations. A cut-off score $(p=0.05)$ of 31 was used for all peptides and since the flagellins of $R$. leguminosarum are highly homologous, we required at least one unique peptide for a flagellin protein to be considered a match. We also determined the relative abundance of the flagellin proteins based on the exponentially modified protein abundance index (emPAI) values, which were automatically generated using MASCOT analysis. The emPAI value is based on the correlation of the observed flagellin peptides in the MS/MS analysis and the number of observable peptides (obtained by in silico digestion) for each flagellin protein $[43,44]$.

\section{Glycoprotein staining}

Flagellar preparations from VF39SM and 3841 were run on $12 \%$ acrylamide at $200 \mathrm{~V}$ for 1 hour and 15 minutes. Glycosylation of flagellin subunits was determined using a Pro-Q Emerald 300 glycoprotein gel stain kit (Molecular Probes) following the manufacturer's instructions. After glycoprotein staining, the total protein was visualized by staining the gel with $0.1 \%$ Coommassie Blue.

\section{Transmission electron microscopy}

Transmission electron microscopy was performed by slightly modifying the procedure used by Miller et al. [28]. The R. leguminosarum wildtype and fla mutant strains were grown on TY plates at $30^{\circ} \mathrm{C}$ for 48 hours. A culture suspension was prepared using sterile double distilled water. A formvar carbon-coated grid was placed on top of a cell suspension drop for 3 minutes and excess liquid was removed. Staining was performed using 1\% uranyl acetate for 30 seconds. Samples were observed using a Philips 410 transmission electron microscope or a Hitachi-7650 transmission electron microscope with images taken with an AMT Image capture Engine. The length of the flagellar filaments formed by the wildtype and mutant strains was measured using Scion Image http://www.scioncorp.com/. 


\section{Results and Discussion}

\section{Characterization of flagellin genes in $R$. leguminosarum}

There are seven flagellin (fla) genes (flaA RL0718, flaB RL0719, flaC RL0720, flaD RL0721, flaE pRL110518, flaH RL3268, and flaG RL4729) in the genome of R. leguminosarum bv. viciae strain 3841 [45]. Sequence analysis and transcriptional studies indicate that all of the seven flagellin genes are transcribed separately as monocistronic genes. Six flagellin genes $($ fla $A / B / C / D / H / G)$ are found on the chromosome, with fla $A / B / C / D$ located within the major chemotaxis and motility gene cluster [28] while flaE is encoded on plasmid pRL11. The sizes of the predicted encoded proteins are: FlaA, 301 amino acids; FlaB, 302 amino acids; FlaC, 303 amino acids; FlaD, 320 amino acids; FlaE, 302 amino acids; FlaH, 337 amino acids, and FlaG, 311 amino acids, respectively. The sizes of these flagellin subunits are smaller than the flagellin proteins of $S$. meliloti (321 to 401 amino acids) $[46,47]$ and $R$. lupini (410-430 amino acids) [5]. The predicted molecular masses of the proteins are: FlaA-31 kDa; FlaB-31 kDa; FlaC-31 kDa; FlaD-34 kDa; FlaE-31; kDa; FlaH-36 kDa; FlaG-32 kDa. Our group has also determined the sequences of the flagellin genes of $R$. leguminosarum strain VF39SM (Genbank accession number GU071045 for flaA/B/C/D; GU071046 for flaE; GU071047 for flaH; and GU071048 for flaG) and found that the predicted flagellin subunits of this strain are $99 \%$ to $100 \%$ identical to the corresponding flagellins in 3841 . All of the flagellin proteins of $R$. leguminosarum exhibit conserved residues at the amino and carboxy-terminal ends (Fig. 1 and 2). The central regions of the proteins, on the other hand, contain the highest variability. In terms of flagellin sequence similarity, FlaA/B/C/E/G are highly similar, exhibiting $86-93 \%$ similarity to each other. The other two flagellins, FlaD and $\mathrm{FlaH}$, are more distant, and respectively share $62 \%$ and $64 \%$ similarity with FlaA.

\section{Ultrastructure of the flagellar filament of $R$. leguminosarum}

Electron microscopy work confirmed that $R$. leguminosarum bv. viciae strain 3841 is subpolarly flagellated [28], while strain VF39SM is peritrichously flagellated, exhibiting 4-7 flagella per cell (Fig. 3). The flagellar filaments of strains 3841 and VF39SM were around $5 \mu \mathrm{m}$ in length and averaged $18 \mathrm{~nm}$ and $17 \mathrm{~nm}$ in width, respectively. The surface of the filaments appeared smooth (Fig. 3c and 3d) and lacked the recognizable cross-hatched pattern observed in the complex flagella of S. meliloti (Fig. 3f) [9,24,26,48] and R. lupini [40]. It is possible that the surface of the $R$. leguminosarum filaments lacks helical perturbations or the perturbations are not as prominent as those of the complex filaments of the other soil bacteria.

\section{Transcription of $\boldsymbol{R}$. leguminosarum fla genes}

Previous transcriptional studies in our lab using gusA fusions demonstrated that for both VF39SM and 3841, flaA, flaC, and flaD have the highest expression (2376 Miller Units (MU) to $6516 \mathrm{MU}$ ) while minimal expression (68 MU to $542 \mathrm{MU}$ ) was observed for flaE, flaH, and fla $G$ [49]. The gene fusion for flaB reported in that paper was made in a different vector, pFAJ1701, so comparisons of flaB expression to that of the other flagellins were not valid. To place levels of flaB transcription in a proper context compared to the other fla genes, a new fusion to the flaB promoter was made in pFus1 (see methods) and gene expression of flaB was measured at $2529 \pm 11 \mathrm{MU}$ in 3841 and $4279 \pm 466$ in VF39SM. These results suggest that flaA, flaB, flaC, and flaD are the major flagellin subunits of R. leguminosarum while flaE, flaH, and flaG play minor roles. However, the presence of post-transcriptional regulation in flagellin biosynthesis cannot be precluded; hence, we performed mutational analysis. We have constructed strains with individual mutations in the seven flagellin genes and two multiple fla mutants $\left(\right.$ fla $B / C / D^{-}$and fla $A / B / C / D^{-}$) for both strains VF39SM and 3841. The resulting mutants were examined for motility defects, using swimming and swarming assays, and morphological defects, using transmission electron microscopy.

\section{Motility assays and electron microscopy of wildtype and fla mutant strains}

The swimming and swarming properties of the wildtype and fla mutant strains are summarized in Table 2. To account for the motility phenotypes of the mutant strains, we determined the effect of mutating the flagellin genes on the structure of the flagellar filament. In general, the flagellar filaments of all the individual flagellin mutants appeared to have normal fine structure and the width of the filament (except VF39SM flaD, which we describe below) was nearly identical to that of the wildtype.

The swimming motility of the 3841 flaA mutant was significantly reduced while the VF39SM flaA mutant was non-motile on swimming plates. Complementation of 3841 flaA and VF39SM flaA mutants with pBBRMCS1-MCS5::flaA completely restored swimming motility, confirming that swimming defects were due to loss of flaA. Both of the flaA mutants were also unable to swarm. The VF39SM flaA mutant strain was non-flagellated (Fig. 4a). Most of the 3841 flaA mutants observed by TEM were non-flagellated. Only one cell was observed to possess a very thin and short appendage (Fig. 5). Individual mutations in flaB for both 3841 and VF39SM, and flaC for VF39SM resulted in a reduced number of flagella and shorter filaments 


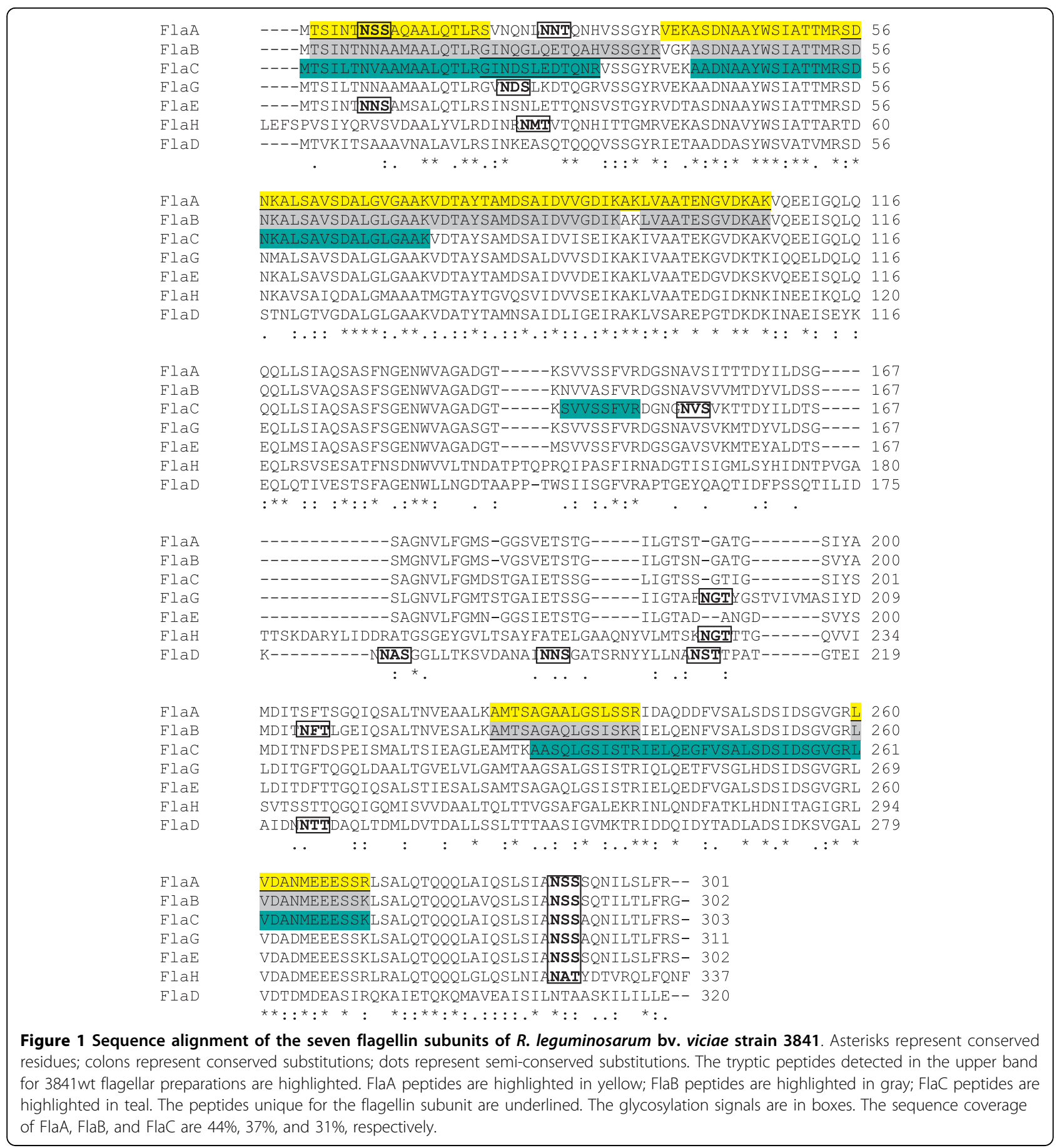

(Fig. 4b and 4c; Fig. 5), which could account for the considerable reduction in swimming and swarming motility (Table 2). The lengths of the flagellar filaments formed by the VF39SM flaB and VF39SM flaC mutants were reduced to around half of the wildtype flagellum. Mutation of flaB in 3841 also resulted in the synthesis of shorter filaments, exhibiting an average length of $2.2 \mu \mathrm{m}$. In terms of the number of filaments formed, almost all of the VF39SM fla $B^{-}$and VF39SM fla $\mathrm{C}^{-}$cells observed exhibited only one flagellum per cell compared with the 4-7 flagella formed by the wildtype strain. Multiple mutations in $f l a A / B / C / D$ for both 3841 and VF39SM (Fig. 4i) resulted in non-flagellated and consequently non-motile strains. Complementation of the 3841 fla $A / B / C / D^{-}$strain with cosmid 976 [50], which was shown by hybridization to carry flaA, flaB, flaC, 
and flaD, restored swimming and swarming motility to near wildtype levels (data not shown). The VF39SM flaE (Fig. 4e), flaH, and flaG mutants (Fig. 4f and 4g) exhibited normal flagellation while VF39SM flaD (Fig. 4d) displayed normal number and length of flagella, although the flagellar filaments were thinner along their entire length (average of $7 \mathrm{~nm}$ width). Also, individual mutations of flaD, flaE, flaH, and flaG did not significantly affect swimming and swarming motility in VF39SM (Table 3). A different phenotype was observed in 3841 flaE and flaH mutants, which exhibited truncated filaments (Fig. 5) and reduced swimming motility. The flagellar filaments formed by the 3841 $\mathrm{flaE}^{-}$and $3841 \mathrm{flaH}^{-}$strains averaged $3.4 \mu \mathrm{m}$ and $2.4 \mu \mathrm{m}$ in length, respectively. Although the swimming motility of 3841 flaE and 3841 flaH mutant strains were reduced, the swarming motility was not significantly affected.

The motility assays and the filament morphologies demonstrate that FlaA is an essential flagellin subunit for $R$. leguminosarum. Mutation of flaA resulted in nonflagellated (for VF39SM) and consequently non-motile 

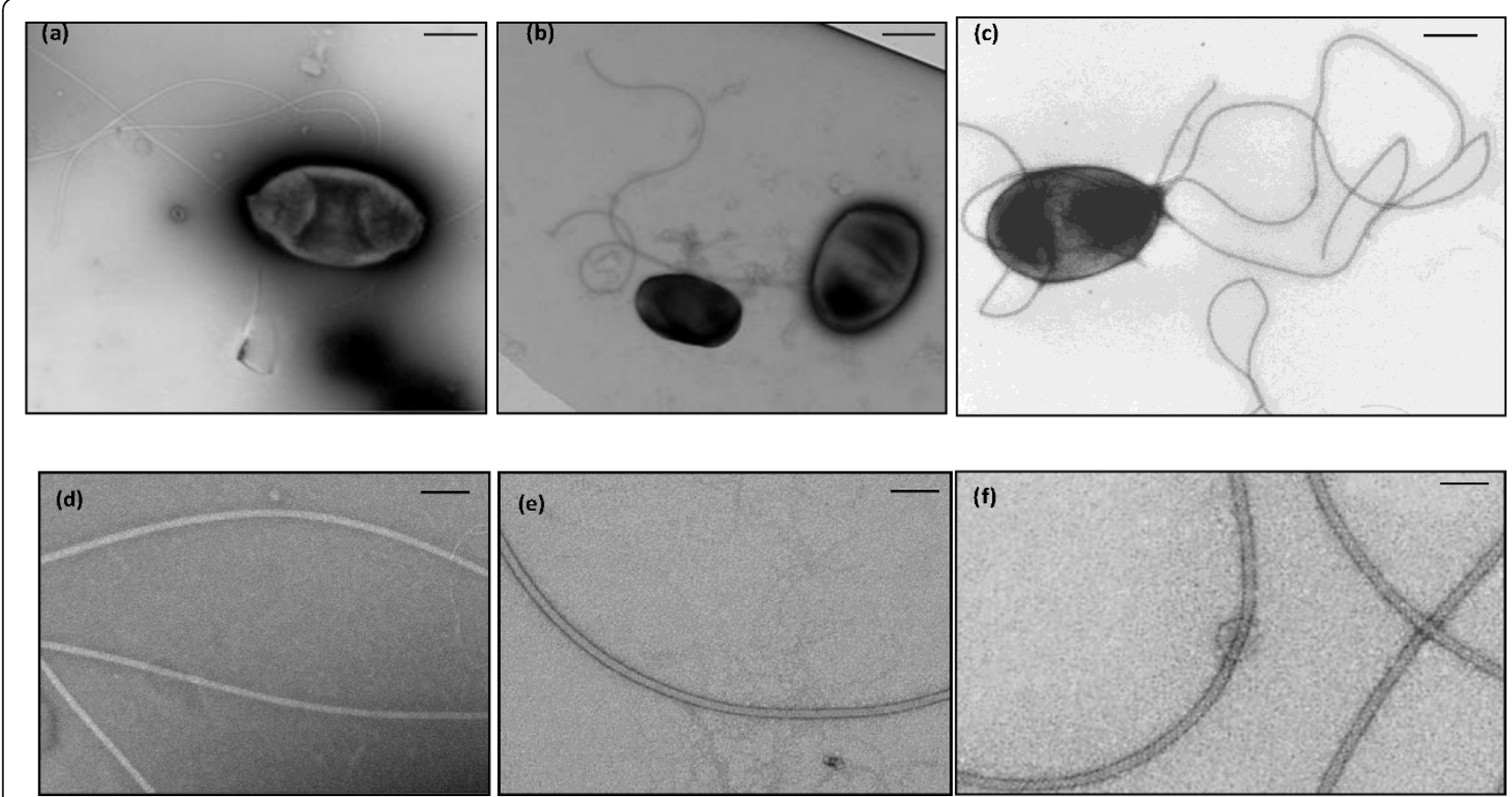

Figure 3 Electron micrographs of $R$. leguminosarum and S. meliloti 1021 flagellar filaments stained with $1 \%$ uranyl acetate. (a) VF39SM is peritrichously flagellated; (b) 3841 has a subpolar flagellum; (c) S. meliloti 1021 is peritrichously flagellated. The flagellar filaments of (d) VF39SM and (e) 3841 appear to have a smooth surface and lack the ridging pattern observed on the surface of the complex flagella formed by ( $f$ ) $S$. meliloti 1021. Bars: $500 \mathrm{~nm}$ for $\mathrm{a}, \mathrm{b}$ and c; $100 \mathrm{~nm}$ for $\mathrm{d}$, e and f.

strains. It is possible that (at least for strain VF39SM), FlaA forms the proximal part of the filament, hence when FlaA is not synthesized, $R$. leguminosarum fails to assemble the distal part of the filaments using the other subunits synthesized. The major role of FlaA in filament assembly and function is similar to what has been reported in S. meliloti, A. tumefaciens, and $R$. lupini $[5,6]$. In all three species, mutation of flaA resulted in non-motile strains. However, unlike the non-flagellated VF39SM flaA mutant, strains of S. meliloti, A. tumefaciens and $R$. lupini with mutations in flaA were able to polymerize severely truncated filaments. Whereas FlaA is an essential subunit, it is not sufficient to assemble a fully functional flagellar filament as demonstrated in the flaB $/ C / D$ mutants. The $f l a B / C / D$ mutant strains exhibited shorter filaments and have reduced numbers of flagella (Table 2), which might have been assembled using FlaA and the other minor flagellin subunits $(\mathrm{FlaE} / \mathrm{H} / \mathrm{G})$. In addition, the assembled filaments were not fully functional as demonstrated by the motility assays.

It is also apparent from our functional studies that both $\mathrm{FlaB}$ and $\mathrm{FlaC}$ are major components of the flagellar filament since mutation in each of the genes resulted in shorter filaments, reduced number of flagella, and consequently reduced motility. It is possible that FlaB and $\mathrm{FlaC}$ are located in the middle part of the filament, hence only the proximal part of the filament, composed of FlaA and possibly other minor subunits, is formed in the flaB and flaC mutants. Additionally, the reduction in the length and number of filaments in the flaB and flaC mutants may reflect an increase in the brittleness and fragility of the filament. Our claim that FlaA, FlaB, and FlaC are the major flagellins of VF39SM and 3841 is further supported by our gene expression studies which demonstrated high promoter activities for flaA, $f l a B$, and flaC. It is also possible that FlaD contributes to the flagellar filament since the amount of flaD transcript was also high and the filaments formed by the VF39SM flaD mutant were thinner than the wildtype. The formation of thinner filaments also suggests that FlaD might be located along the entire length of the filament for VF39SM, thus the need for a high amount of flaD transcripts. However, it is remarkable that the swimming and swarming motility of the VF39SM flaD mutant are not impaired. A possible explanation could be that the width of the filament formed by the flaD mutant is still enough to support the normal function of the flagella. Contrary to the major roles of $\mathrm{FlaA} / \mathrm{B} / \mathrm{C} / \mathrm{D}$ in VF39SM, FlaE, FlaH, and FlaG appear to be minor components of the flagellar filament as indicated by expression levels as measured in gene fusions, and by the subtle effects of their mutations on flagellar filament morphology and on motility. In 3841, FlaE and FlaH appeared to be important for swimming but not for 
Table 2 Properties of $\boldsymbol{R}$. leguminosarumwildtype and flagellin mutants

\begin{tabular}{|c|c|c|c|c|}
\hline Strain/Mutant & $\begin{array}{l}\text { Effective Fla } \\
\text { subunit(s) }\end{array}$ & $\begin{array}{l}\text { Swimming } \\
\text { diameter* }\end{array}$ & $\begin{array}{c}\text { Swarming } \\
\text { ability* }\end{array}$ & Filament Morphology $\dagger$ \\
\hline Strain 3841 & ABCDEHG & 100 & +++ & Normal $(4.7 \pm 0.5 u m ; n=8)$ \\
\hline $3841 \mathrm{flaA}^{-}$ & BCDEHG & 8 & - & $\begin{array}{l}\text { Almost all cells are non-flagellated; only one cell with very thin, short } \\
\text { appendage }\end{array}$ \\
\hline $3841 \mathrm{flaB}^{-}$ & ACDEHG & 47 & + & Truncated $(2.2 \pm 0.5 u m ; n=6)$ \\
\hline $3841 \mathrm{flaC}$ & ABDEHG & 30 & ++ & ND \\
\hline $3841 \mathrm{flaD}^{-}$ & ABCEHG & 87 & +++ & ND \\
\hline 3841 flaE & $\mathrm{ABCDHG}$ & 39 & ++++ & Truncated (3.4 \pm 0.3 um; $n=5)$ \\
\hline $3841 \mathrm{flaH}^{-}$ & ABCDEG & 54 & +++ & Truncated $(2.4 \pm 0.6$ um; $n=12)$ \\
\hline $3841 \mathrm{flaG}$ & ABCDEH & 96 & ++ & ND \\
\hline $3841 \mathrm{flaB} / \mathrm{C}^{-} \mathrm{D}^{-}$ & AEHG & 26 & + & Truncated $(1.9 \pm 0.6$ um; $n=13)$ \\
\hline $3841 \mathrm{flaA} / \mathrm{B} / \mathrm{C} / \mathrm{D}^{-}$ & EHG & - & - & ND \\
\hline Strain VF39SM & ABCDEHG & 100 & +++++ & Normal $(5.1 \pm 0.5$ um; $n=13)$ \\
\hline VF39SM flaA- & BCDEHG & - & - & No flagella \\
\hline VF39SM flaB- & ACDEHG & 41 & ++ & $\begin{array}{l}\text { Truncated }(1.6 \pm 0.5 \text { um; } n=6) \text {; reduced number of filaments }(1-2 \\
\text { filaments/cell) }\end{array}$ \\
\hline VF39SM flaC & ABDEHG & 49 & ++ & $\begin{array}{l}\text { Truncated }(2.1 \pm 0.5 \text { um; } n=9) \text {; reduced number of filaments }(1-2 \\
\text { filaments/cell) }\end{array}$ \\
\hline VF39SM flaD & ABCEHG & 85 & ++++ & Normal number and length; thinner filaments \\
\hline VF39SM flaE & $\mathrm{ABCDHG}$ & 92 & ++++ & Normal \\
\hline VF39SM flaH & ABCDEG & 97 & +++++ & Normal \\
\hline VF39SM flaG & ABCDEH & 100 & +++ & Normal; slightly reduced number of filaments \\
\hline $\begin{array}{l}\text { VF39SM flaB/Cl } \\
D^{-}\end{array}$ & AEHG & 25 & + & $\begin{array}{l}\text { Truncated }(1.6 \pm 0.3 \text { um; } n=13) \text {; reduced number of filaments }(1-2 \\
\text { filaments/cell) }\end{array}$ \\
\hline $\begin{array}{l}\text { VF39SM flaA/B/ } \\
C / D^{-}\end{array}$ & EHG & - & - & No flagella \\
\hline
\end{tabular}

*Percentage relative to wildtype swimming diameter. Means of at least two replicates. (-) means non-motile; + As observed by TEM; ND means not determined; values in parenthesis refer to the average length of a flagellar filament \pm standard deviation. The lengths of the flagella formed by the fla mutants are significantly different from the flagella formed by the wildtype strain $(P<0.0001)$.

swarming motility. Since the TEM images for the wildtype and fla mutant strains were obtained from vegetative cells, it would be interesting to observe the filaments formed by the swarm cells of $3841 \mathrm{flaE}$ and 3841 flaH mutants.

\section{Tandem mass spectrometry analysis}

Flagellar samples were prepared from the wildtype strains and were run on SDS-PAGE. Immunoblots were prepared using a polyclonal flagellar antibody. However, due to the similar size of all seven flagellins $(31-36 \mathrm{kDa})$, we failed to resolve all subunits (Additional file 3). Thus, we decided to perform tandem mass spectrometry analysis to identify the flagellin subunits that are incorporated by the wildtype strains into flagellar filaments. We frequently observed two adjacent bands in the protein gel for both 3841 and VF39SM (see fig. 6 for VF39SM). To determine the subunits present in each of the two bands, the bands were analyzed separately for 3841. For VF39SM, the two bands were pooled together. Using the mass spectrometry data, we were also able to estimate the relative abundance of the flagellin subunits using the emPAI values [43]. It has been shown in a previous study that the emPAI value is directly proportional to protein content [44] and this parameter has been utilized in determining the relative abundance of a number of proteins [51-54]. The emPAI value provides an easy estimate of protein abundance since it is automatically generated using the Mascot program.

The locations of the flagellin peptides detected in the flagellar preparations are indicated in Fig. 1 and 2. Only FlaA, FlaB, and FlaC peptides were detected in the flagellar preparation for strain 3841 (for both the lower and the upper bands; Table 3) with sequence coverage ranging from $31 \%$ to $46 \%$. These three subunits also comprised the majority of the flagellin subunits detected in VF39SM (Table 3). FlaE and FlaG comprised a small fraction of the flagellin subunits detected in the VF39SM wt strain. The sequence coverage for the flagellin subunits detected in VF39SM ranged from $18 \%$ to $46 \%$. The results obtained from the MS/MS analysis indicate that at least three flagellin subunits $(\mathrm{FlaA} / \mathrm{B} / \mathrm{C})$ are incorporated into the functional flagellar filament of strain 3841 while VF39SM polymerizes five flagellins 

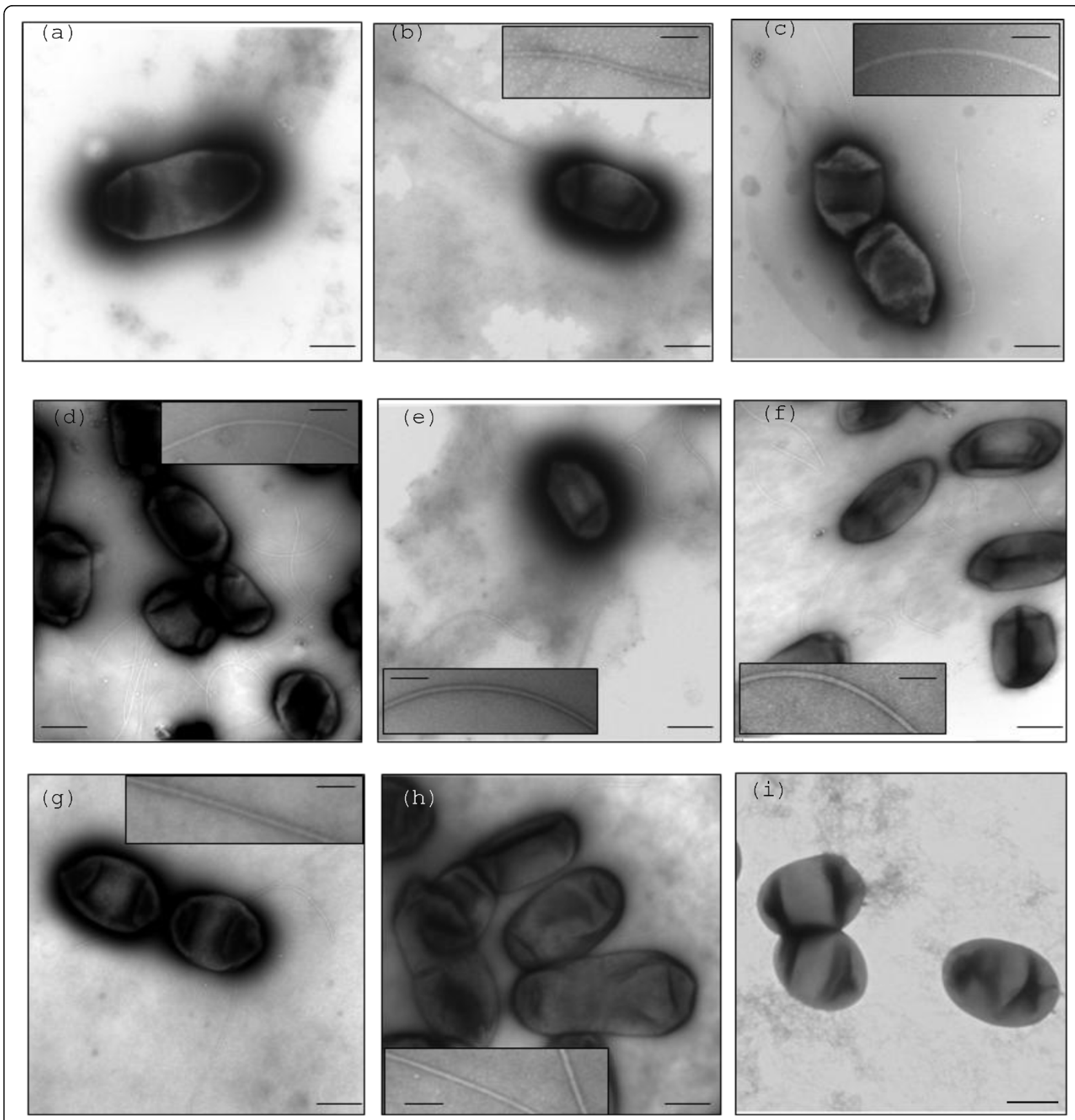

Figure 4 Electron micrographs of $\boldsymbol{R}$. leguminosarum VF39SM fla mutants stained with uranyl acetate. Inset pictures show the flagellar filaments at higher magnification. (a) flaA- (b) flaB- (c) flaC (d) flaD- (e) flaE (f) flaH (g) flaG (h) flaB/C/D' (i) flaA/B/C/D. Bars: $500 \mathrm{~nm}$ for cells with flagella; $100 \mathrm{~nm}$ for inset pictures.

(FlaA/B/C/E/G) into its flagellar filament. The consistently shorter flagellar filaments formed by the flagellin mutants (VF39SM/3841 flaB and flaC mutants) and the absence of flagellar filaments in VF39SM flaA mutants and nearly all cells of $3841 \mathrm{flaA}^{-}$also suggest that the major subunits (FlaA, FlaB, and FlaC), at least, are present in the complete flagella that are assembled.
Peptides for FlaD, FlaE, FlaH, and FlaG were not detected in the flagellar preparation for 3841 while FlaD peptides were not detected in VF39SM. The absence of the flagellin subunits could be due to the following reasons: (a) they are not synthesized under the conditions tested; (b) the subunits are synthesized but at a very low concentration, hence they remained undetected; and/or 

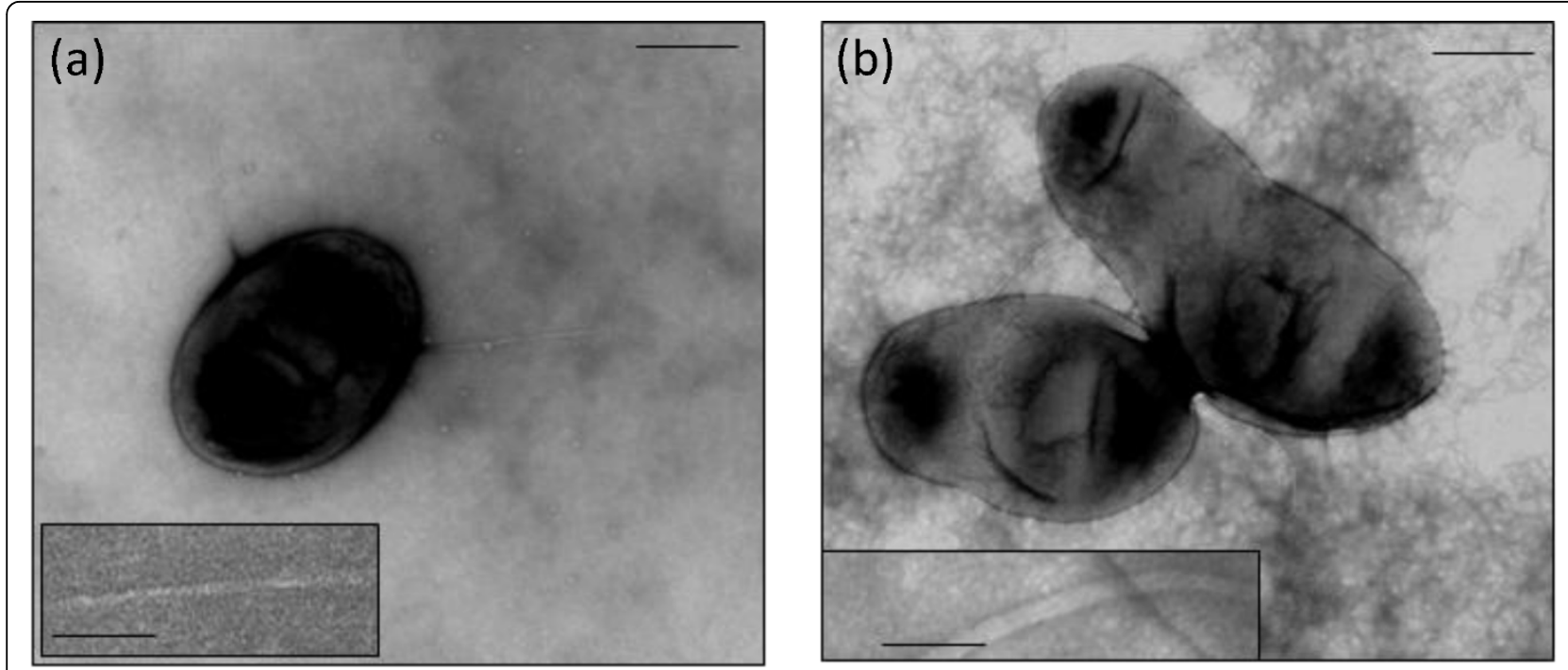

\section{(c)}
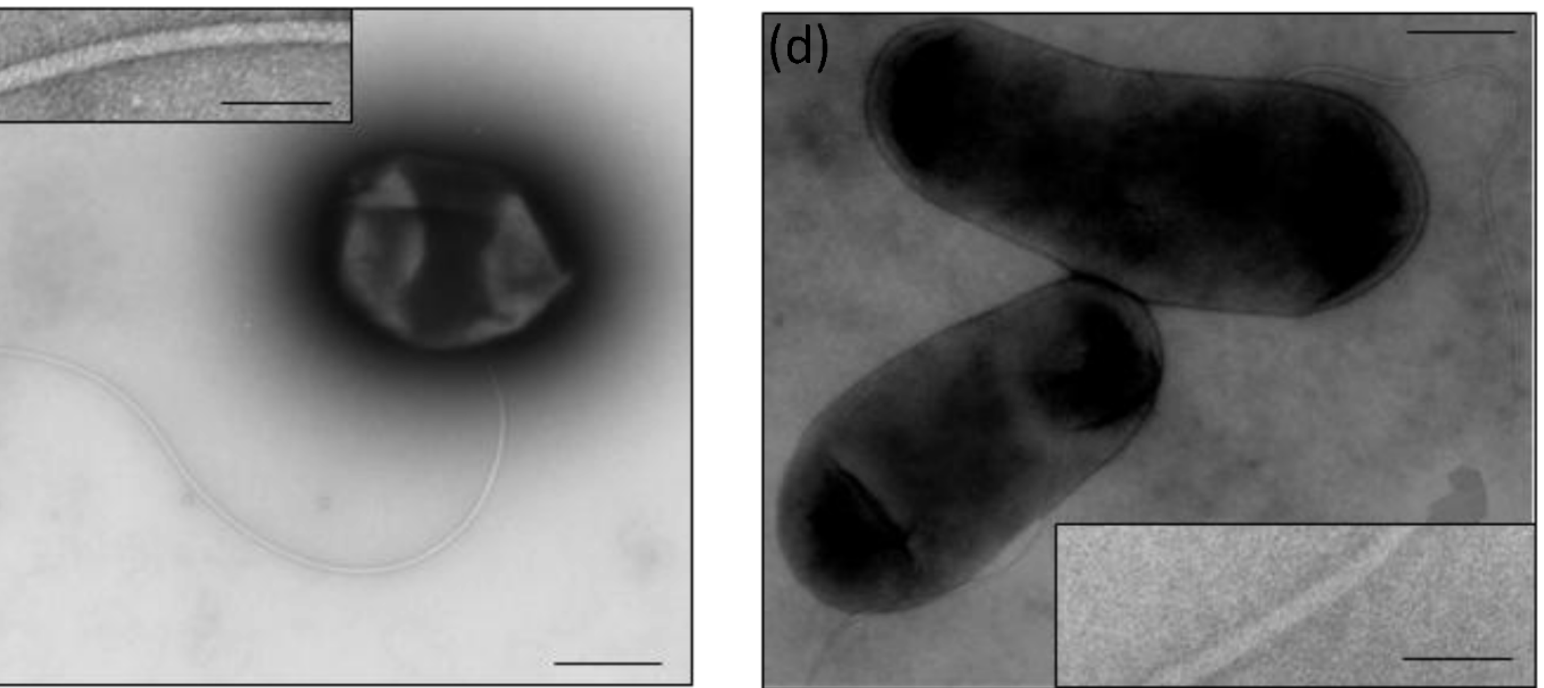

Figure 5 Electron micrographs of $\boldsymbol{R}$. leguminosarum $\mathbf{3 8 4 1}$ fla mutants stained with uranyl acetate. Inset pictures show the flagellar filaments at higher magnification. (a) flaA- (b) flaB- (c) flaE (d) flaH Bars: $500 \mathrm{~nm}$ for cells with flagella; $100 \mathrm{~nm}$ for inset pictures.

(c) the flagellin subunits are highly unstable. For strain 3841, mutation of flaE and flaH resulted in a reduction in swimming motility, suggesting that these subunits probably contribute to the flagellar filament. However, FlaE and FlaH peptides were not detected in the wildtype flagellar preparations, indicating that these peptides may not be stable under the conditions used.

\section{Glycosylation of flagellin subunits}

We observed that for strain 3841, both the upper and the lower bands on the protein gel contained the same set of flagellin subunits (FlaA, FlaB, and FlaC) (Table 3). The molecular masses (around $35 \mathrm{kDa}$; Additional file 3) of the bands observed on the gel also appeared to be higher than the predicted molecular masses $(31 \mathrm{kDa})$ for FlaA and FlaB. This suggests that at least FlaA and FlaB may have undergone post-translational modification, resulting in a higher molecular weight and subsequently slower migration in the protein gel.

Analysis of the flagellin amino acid sequences of R. leguminosarum (Fig. 1 \&2) revealed the presence of two to four putative glycosylation signals $(\mathrm{N}-\mathrm{X}-\mathrm{S} / \mathrm{T}$, where $\mathrm{X}$ is any amino acid except proline) [55]. The MS/MS spectral data for the identified peptides containing the glyosylation signal were also analyzed for the presence of glycosylation, based on the presence of peaks $(\mathrm{m} / \mathrm{z})$ corresponding to different types of glycosylation (Additional file 4 shows a sample of a MS/MS 
Table 3 Flagellin subunits and their relative abundance in $R$. leguminosarum wildtype strains based on tandem mass spectrometry analysis

\begin{tabular}{|c|c|c|c|c|c|}
\hline Flagellin subunit & Queries Matched & No. of unique peptides detected & Sequence coverage (\%) & emPAl & Mascot score \\
\hline \multicolumn{6}{|c|}{ A. 3841 wt lower band } \\
\hline FlaB & 21 & 4 & 42 & 5.85 & 856 \\
\hline FlaA & 19 & 5 & 46 & 4.66 & 622 \\
\hline FlaC & 12 & 2 & 41 & 1.46 & 401 \\
\hline \multicolumn{6}{|c|}{ B. 3841 wt upper band } \\
\hline FlaB & 22 & 4 & 37 & 4.05 & 741 \\
\hline$\overline{\text { FlaA }}$ & 19 & 7 & 44 & 3.62 & 493 \\
\hline FlaC & 13 & 3 & 31 & 1.23 & 288 \\
\hline \multicolumn{6}{|l|}{ A. VF39SM wt } \\
\hline FlaB & 36 & 5 & 43 & 8.28 & 1116 \\
\hline FlaA & 24 & 8 & 46 & 6.68 & 748 \\
\hline FlaG & 16 & 2 & 28 & 2.25 & 415 \\
\hline FlaC & 18 & 2 & 29 & 1.72 & 469 \\
\hline FlaE & 10 & 1 & 18 & 0.83 & 264 \\
\hline
\end{tabular}

spectrum). However, we have not identified any potential glycosylation for these peptides which may be attributed to the lability of this modification [56,57]. Also, sequence coverage only ranged from $18 \%$ to $46 \%$ (Fig. 1 and 2) and peptides at the C-termini of the flagellin subunits were not detected. The $\mathrm{C}$-terminus contains a common glycosylation site for the $R$. leguminosarum flagellin subunits but these glycosylations were not detected in the MS/MS analysis, which could be due to the above reason. Thus, we performed glycoprotein staining to determine if the flagellins are post-translationally modified by glycosylation. We observed positive staining for the flagellins of both VF39SM and 3841 suggesting that these flagellins are glycosylated (Fig. 6). We were unable to determine which flagellins are glycosylated because the seven flagellins were not separated on the protein gel. Glycosylation of flagellins has been reported in a number of animal and plant pathogens including Campylobacter jejuni [56,57], Helicobacter pylori [57,58], Pseudomonas aeruginosa [59,60],

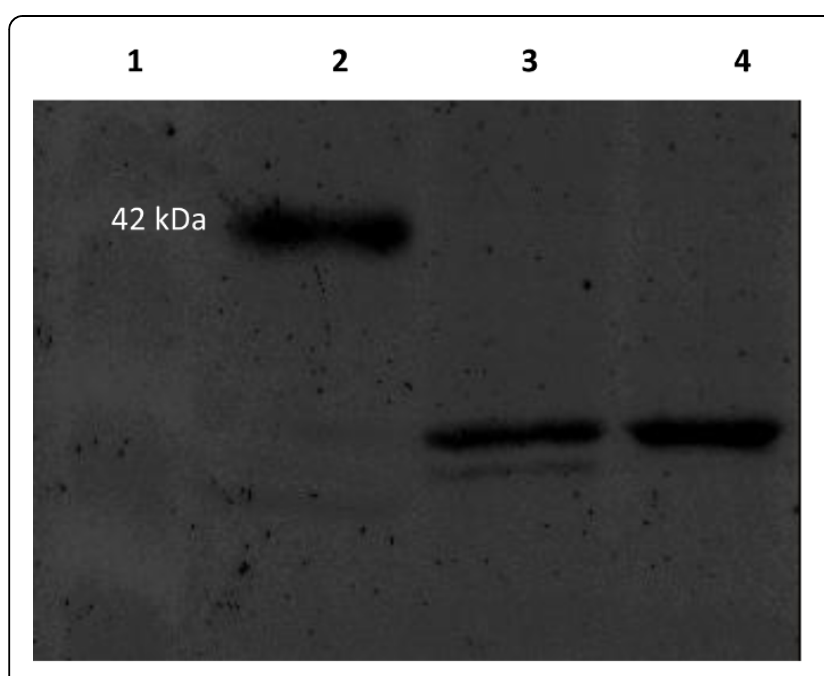

(A)

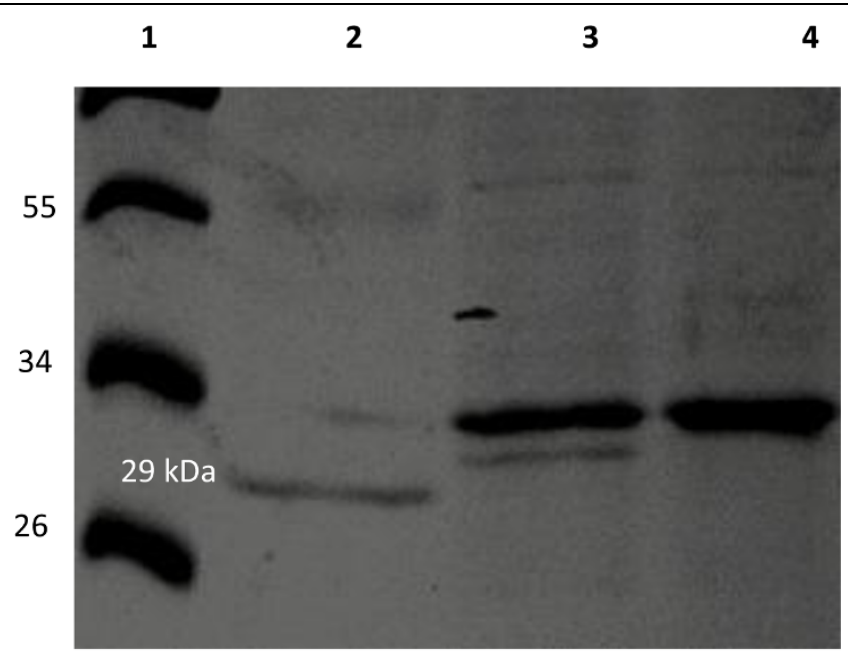

(B)

Figure 6 Glycoprotein staining of $\boldsymbol{R}$. leguminosarum flagellin proteins. A. Pro-Q Emerald 300 stain. Lane 1-Molecular marker. Molecular masses (in kDa) are shown on the left of panel B; Lane 2-CandyCane glycoprotein molecular weight standard, 42kDa $\alpha 1$-Acid glycoprotein served as a positive control (shown in panel A) and a 29kDa-protein, carbonic anhydrase (shown in panel B) served as a negative control for glycosylation; Lane 3 - VF39SM; Lane 4 - 3841. B. Coomassie Brilliant Blue stain to demonstrate total proteins. Same sample arrangement as in panel A. 
Pseudomonas syringae [61,62], Listeria monocytogenes [63,64], A. tumefaciens [6], Acidovorax avenae [65], as well as in the nitrogen-fixing bacterium Azosprillum brasilense [66]. It has been suggested that glycosylation may play a role in flagellar filament assembly and in pathogenesis $[67,68]$. In soil bacteria, it may also function in the attachment of bacteria to the plant roots [66], and in avoiding recognition by the host plant [61].

\section{Conclusions}

In this study, we were able to clarify the roles of the seven flagellin subunits in the assembly of the flagellar filament in $R$. leguminosarum. Taken altogether, our results indicate that FlaA is an essential subunit, but that it is not enough to assemble a fully functional flagellar filament. $\mathrm{FlaB}$ and $\mathrm{FlaC}$ are major components of the filament while FlaD, FlaE, FlaH, and FlaG are only minor components. To assemble a fully functional filament, at least three (FlaA, FlaB, and $\mathrm{FlaC}$ ) and five (FlaA, FlaB, FlaC, FlaE, and FlaG) flagellin subunits should be synthesized by 3841 and VF39SM, respectively. There were no substantial differences in the requirements for individual flagellins in swimming vs. swarming motility. The flagellins of 3841 and VF39SM are possibly modified by glycosylation.

\section{Additional material}

Additional file 1: Sequences of primers used to PCR amplify

flagellin genes. Table showing PCR primer sequences for all PCR work

discussed in the paper.

Additional file 2: Details of flagellin gene mutations in $R$. leguminosarum strains $\mathbf{3 8 4 1}$ and VF39SM. Table giving complete description of fragments and cassettes used in construction of all the mutants described in the paper.

Additional file 3: Immunoblot using an anti-flagellar antibody against flagellar preparations of $\boldsymbol{R}$. leguminosarum. Figure showing western blot of flagellar preparations of wild type and mutant strains.

Additional file 4: MS/MS spectrum of one tryptic peptide from the data set for VF39SM. Figure showing a Mass Spectrum of a peptide from the tryptic digest of VF39SM flagellar proteins.

\section{List of Abbreviations}

MS: mass spectrometry; MS/MS: tandem mass spectrometry; LC: liquid chromatography; PBS: phosphate-buffered saline; emPAl: exponentially modified protein abundance index; TEM: transmission electron microscopy; SDS-PAGE: Sodium-dodecyl sulfate polyacrylamide gel electrophoresis.

\section{Acknowledgements}

We gratefully acknowledge the support for this work from Natural Sciences and Engineering Research Council of Canada (NSERC) Discovery Grants to MFH and SFK. DDT was supported by a Government of Canada graduate scholarship and the Bettina Bahlsen scholarship. We thank Carol Stremick for her help with the protein work as well as Wei-Xiang Dong at the Microscopy and Imaging Facility of the University of Calgary for his assistance with electron microscopy.

We also thank Dr. Christopher K. Yost for his very helpful comments on the manuscript.

\section{Author details}

${ }^{1}$ Department of Biological Sciences, University of Calgary. 2500 University Drive NW. Calgary, AB, T2N 1N4, Canada. ${ }^{2}$ Department of Microbiology and Immunology, University of Western Ontario, London, Ontario, N6A 5C1, Canada. ${ }^{3}$ Mass Spectrometry Facility, Faculty of Medicine, University of Calgary. 2500 University Drive NW. Calgary, AB, T2N 1N4, Canada.

\section{Authors' contributions}

DDT was involved in the design of the study and in carrying out the experiments. DDT also prepared the draft for the manuscript. DEB and KLD were involved in conducting the experiments, which included construction of the mutants and gusA fusion strains and gusA assays. SFK was involved in the TEM work for the wildtype strains and some VF39SM mutants, and has been involved in revising the manuscript. MFK participated in interpreting the MS/MS results. MFH conceived the study, supervised the experiments, and was involved in writing and finalizing the manuscript. All authors read and approved the final manuscript.

Received: 10 April 2010 Accepted: 17 August 2010

Published: 17 August 2010

\section{References}

1. Silverman M: Building bacterial flagella. Q Rev Biol 1980, 55(4):395-408,

2. Macnab RM: How bacteria assemble flagella. Annu Rev Microbiol 2003, 57(1):77-100.

3. Enomoto M, Sakai A, Tominaga A: Expression of an Escherichia coli flagellin gene, hag48, in the presence of a Salmonella H1-repressor. Mol Gen Genet 1985, 201(1):133-135.

4. Kuwajima G, Asaka J, Fujiwara T, Node K, Kondo E: Nucleotide sequence of the hag gene encoding flagellin of Escherichia coli. J Bacteriol 1986, 168(3):1479-1483.

5. Scharf $B$, Schuster-Wolff-Buhring $H$, Rachel $R$, Schmitt R: Mutational analysis of the Rhizobium lupini $\mathrm{H} 13-3$ and Sinorhizobium meliloti flagellin genes: importance of flagellin A for flagellar filament structure and transcriptional regulation. J Bacteriol 2001, 183:5334-5342.

6. Deakin WJ, Parker VE, Wright EL, Ashcroft KJ, Loake GJ, Shaw CH: Agrobacterium tumefaciens possesses a fourth flagelin gene located in a large gene cluster concerned with flagellar structure, assembly and motility. Microbiology 1999, 145(Pt 6):1397-1407.

7. Chesnokova O, Coutinho JB, Khan IH, Mikhail MS, Kado Cl: Characterization of flagella genes of Agrobacterium tumefaciens, and the effect of a bald strain on virulence. Mol Microbiol 1997, 23(4):579-590.

8. Götz R, Limmer N, Ober K, Schmitt R: Motility and chemotaxis in two strains of Rhizobium with complex flagella. J Gen Microbiol 1982 128(4):789-798.

9. Pleier E, Schmitt R: Expression of two Rhizobium meliloti flagellin genes and their contribution to the complex filament structure. J Bacterio/ 1991 173(6):2077-2085.

10. Bergman K, Nulty E, Su LH: Mutations in the two flagellin genes of Rhizobium meliloti. J Bacteriol 1991, 173(12):3716-3723.

11. Cohen-Krausz S, Trachtenberg S: The axial alpha-helices and radial spokes in the core of the cryo-negatively stained complex flagellar filament of Pseudomonas rhodos: recovering high-resolution details from a flexible helical assembly. J Mol Biol 2003, 331(5):1093-1108.

12. Cohen-Krausz S, Trachtenberg S: Helical perturbations of the flagellar filament: Rhizobium lupini $\mathrm{H} 13-3$ at 13 A resolution. J Struct Biol 1998, 122(3):267-282.

13. Namba K, Yamashita I, Vonderviszt F: Structure of the core and central channel of bacterial flagella. Nature 1989, 342(6250):648-654.

14. Samatey FA, Imada K, Nagashima S, Vonderviszt F, Kumasaka T, Yamamoto M, Namba K: Structure of the bacterial flagellar protofilament and implications for a switch for supercoiling. Nature 2001, 410(6826):331-337

15. Mimori Y, Yamashita I, Murata K, Fujiyoshi Y, Yonekura K, Toyoshima C Namba K: The structure of the R-type straight flagellar filament of Salmonella at 9 A resolution by electron cryomicroscopy. J Mol Biol 1995, 249(1):69-87

16. Mimori-Kiyosue $Y$, Yamashita I, Fujiyoshi $Y$, Yamaguchi S, Namba K: Role of the outermost subdomain of Salmonella flagellin in the filament structure revealed by electron cryomicroscopy. J Mol Biol 1998, 284(2):521-530. 
17. Morgan DG, Owen C, Melanson LA, DeRosier DJ: Structure of bacterial flagellar filaments at $11 \mathrm{~A}$ resolution: packing of the alpha-helices. $\mathrm{J} \mathrm{Mol}$ Biol 1995, 249(1):88-110.

18. Hyman HC, Trachtenberg S: Point mutations that lock Salmonella typhimurium flagellar filaments in the straight right-handed and lefthanded forms and their relation to filament superhelicity. $J \mathrm{Mol}$ Biol 1991, 220(1):79-88.

19. Mimori-Kiyosue $Y$, Vonderviszt F, Namba K: Locations of terminal segments of flagellin in the filament structure and their roles in polymerization and polymorphism. J Mol Biol 1997, 270(2):222-237.

20. Trachtenberg S, DeRosier DJ: Three-dimensional reconstruction of the flagellar filament of Caulobacter crescentus. A flagellin lacking the outer domain and its amino acid sequence lacking an internal segment. $J \mathrm{Mol}$ Biol 1988, 202(4):787-808.

21. Yoshioka K, Aizawa S, Yamaguchi S: Flagellar filament structure and cell motility of Salmonella typhimurium mutants lacking part of the outer domain of flagellin. J Bacterio/ 1995, 177(4):1090-1093.

22. Kuwajima G: Construction of a minimum-size functional flagellin of Escherichia coli. J Bacteriol 1988, 170(7):3305-3309.

23. Cohen-Krausz S, Trachtenberg S: The structure of the helically perturbed flagellar filament of Pseudomonas rhodos: implications for the absence of the outer domain in other complex flagellins and for the flexibility of the radial spokes. Mol Microbiol 2003, 48(5):1305-1316.

24. Trachtenberg S, DeRosier DJ, Macnab RM: Three-dimensional structure of the complex flagellar filament of Rhizobium lupini and its relation to the structure of the plain filament. J Mol Biol 1987, 195(3):603-620.

25. Schmitt R, Raska I, Mayer F: Plain and complex flagella of Pseudomonas rhodos: analysis of fine structure and composition. J Bacterio/ 1974, 117(2):844-857.

26. Krupski G, Götz R, Ober K, Pleier E, Schmitt R: Structure of complex flagellar filaments in Rhizobium meliloti. J Bacteriol 1985, 162(1):361-366.

27. Trachtenberg S, Hammel I: The rigidity of bacterial flagellar filaments and its relation to filament polymorphism. J Struct Biol 1992, 109(1):18-27.

28. Miller LD, Yost CK, Hynes MF, Alexandre G: The major chemotaxis gene cluster of Rhizobium leguminosarum bv. viciae is essential for competitive nodulation. Mol Microbiol 2007, 63(2):348-362

29. Tambalo DD, Yost CK, Hynes MF: Characterization of swarming motility in Rhizobium leguminosarum biovar viciae. FEMS Microbiol Lett 2010, 307:165-174.

30. Beringer JE: R factor transfer in Rhizobium leguminosarum. J Gen Microbiol 1974, 84(1):188-198

31. Sambrook J, Fritsch EF, Maniatis T: Molecular cloning-A laboratory manual New York: Cold Sping Harbor, 21989.

32. Quandt J, Hynes MF: Versatile suicide vectors which allow direct selection for gene replacement in Gram-negative bacteria. Gene 1993, 127(1):15-21

33. Reeve WG, Tiwari RP, Worsley PS, Dilworth MJ, Glenn AR, Howieson JG: Constructs for insertional mutagenesis, transcriptional signal localization and gene regulation studies in root nodule and other bacteria. Microbiology 1999, 145:1307-1316.

34. Prentki $P$, Krisch HM: In vitro insertional mutagenesis with a selectable DNA fragment. Gene 1984, 29(3):303-313.

35. Fellay R, Frey J, Krisch $\mathrm{H}$ : Interposon mutagenesis of soil and water bacteria: a family of DNA fragments designed for in vitro insertional mutagenesis of Gram-negative bacteria. Gene 1987, 52(2-3):147-154.

36. Alexeyev MF, Shokolenko IN, Croughan TP: Improved antibiotic-resistance gene cassettes and omega elements for Escherichia coli vector construction and in vitro deletion/insertion mutagenesis. Gene 1995, 160(1):63-67.

37. Jefferson RA, Burgess SM, Hirsh D: Beta-glucuronidase from Escherichia coli as a gene-fusion marker. Proc Natl Acad Sci USA 1986, 83(22):8447-8451.

38. Yost CK, Del Bel KL, Quandt J, Hynes MF: Rhizobium leguminosarum methyl-accepting chemotaxis protein genes are down-regulated in the pea nodule. Arch Microbiol 2004, 182(6):505-513.

39. Ames $P$, Schluederberg SA, Bergman K: Behavioral mutants of Rhizobium meliloti. J Bacteriol 1980, 141(2):722-727.

40. Maruyama M, Lodderstaedt G, Schmitt R: Purification and biochemical properties of complex flagella isolated from Rhizobium lupini $\mathrm{H} 13-3$. Biochim Biophys Acta 1978, 535(1):110-124

41. Del Bel KL: Genetic regulation of chemotaxis and motility in Rhizobium leguminosarum. microform Calgary: Thesis, University of Calgary 2004.
42. Deutscher MP: Guide to protein purification. San Diego, Calif:: Academic Press 1990.

43. Ishihama Y, Oda Y, Tabata T, Sato T, Nagasu T, Rappsilber J, Mann M: Exponentially modified protein abundance index (emPAl) for estimation of absolute protein amount in proteomics by the number of sequenced peptides per protein. Mol Cell Proteomics 2005, 4(9):1265-1272.

44. Ishihama Y, Schmidt T, Rappsilber J, Mann M, Hartl FU, Kerner MJ, Frishman D: Protein abundance profiling of the Escherichia coli cytosol. BMC Genomics 2008, 9:102

45. Young JPW, Crossman LC, Johnston AW, Thomson NR, Ghazoui ZF, Hull KH, Wexler M, Curson AR, Todd JD, Poole PS, et al: The genome of Rhizobium leguminosarum has recognizable core and accessory components. Genome Biol 2006, 7(4):R34

46. Capela D, Barloy-Hubler F, Gouzy J, Bothe G, Ampe F, Batut J, Boistard P,

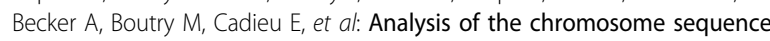
of the legume symbiont Sinorhizobium meliloti strain 1021. Proc Natl Acad Sci USA 2001, 98(17):9877-9882.

47. Pleier $E$, Schmitt $R$ : Identification and sequence analysis of two related flagellin genes in Rhizobium meliloti. J Bacteriol 1989, 171(3):1467-1475.

48. Trachtenberg S, DeRosier DJ: Three-dimensional structure of the frozenhydrated flagellar filament: The left-handed filament of Salmonella typhimurium. J Mol Biol 1987, 195(3):581-601.

49. Tambalo DD, Del Bel KL, Bustard DE, Greenwood PR, Steedman AE, Hynes MF: Regulation of flagellar, motility and chemotaxis genes in Rhizobium leguminosarum by the VisN/R-Rem cascade. Microbiology 2010, 156:1673-1685

50. Yost CK: Characterization of Rhizobium leguminosarum genes homologous to chemotaxis chemoreceptors. microform Calgary: Thesis, University of Calgary 1998.

51. Mann K, Poustka A, Mann M: In-depth, high-accuracy proteomics of sea urchin tooth organic matrix. Proteome Sci 2008, 6(1):33.

52. Borsuk S, Newcombe J, Mendum TA, Dellagostin OA, McFadden J: Identification of proteins from tuberculin purified protein derivative (PPD) by LC-MS/MS. Tuberculosis 2009, 89(6):423-430.

53. Gold ND, Martin VJJ: Global view of the Clostridium thermocellum cellulosome revealed by quantitative proteomic analysis. J Bacterio/ 2007, 189(19):6787-6795.

54. Mastroleo F, Leroy B, Van Houdt R, s' Heeren C, Mergeay M, Hendrickx L, Wattiez R: Shotgun proteome analysis of Rhodospirillum rubrum $\mathrm{S} 1 \mathrm{H}$ : integrating data from gel-free and gel-based peptides fractionation methods. J Proteome Res 2009, 8(5):2530-2541.

55. Gavel Y, von Heijne G: Sequence differences between glycosylated and non-glycosylated Asn-X-Thr/Ser acceptor sites: implications for protein engineering. Protein Eng 1990, 3(5):433-442.

56. Thibault $P$, Logan SM, Kelly JF, Brisson JR, Ewing CP, Trust TJ, Guerry P: Identification of the carbohydrate moieties and glycosylation motifs in Campylobacter jejuni flagellin. J Biol Chem 2001, 276(37):34862-34870.

57. Schirm M, Schoenhofen IC, Logan SM, Waldron KC, Thibault P: Identification of unusual bacterial glycosylation by tandem mass spectrometry analyses of intact proteins. Anal Chem 2005, 77(23):7774-7782.

58. Schirm M, Soo EC, Aubry AJ, Austin J, Thibault P, Logan SM: Structural, genetic and functional characterization of the flagellin glycosylation process in Helicobacter pylori. Mol Microbiol 2003, 48(6):1579-1592.

59. Arora SK, Bangera M, Lory S, Ramphal R: A genomic island in Pseudomonas aeruginosa carries the determinants of flagellin glycosylation. Proc Natl Acad Sci USA 2001, 98(16):9342-9347.

60. Schirm M, Arora SK, Verma A, Vinogradov E, Thibault P, Ramphal R, Logan SM: Structural and genetic characterization of glycosylation of type a flagellin in Pseudomonas aeruginosa. J Bacteriol 2004, 186(9):2523-2531.

61. Taguchi F, Takeuchi K, Katoh E, Murata K, Suzuki T, Marutani M, Kawasaki T, Equchi M, Katoh S, Kaku H, et al: Identification of glycosylation genes and glycosylated amino acids of flagellin in Pseudomonas syringae pv. tabaci. Cell Microbiol 2006, 8(6):923-938.

62. Takeuchi K, Taguchi F, Inagaki Y, Toyoda K, Shiraishi T, Ichinose Y: Flagellin glycosylation island in Pseudomonas syringae pv. glycinea and its role in host specificity. J Bacteriol 2003, 185(22):6658-6665.

63. Shen A, Kamp HD, Grundling A, Higgins DE: A bifunctional O-GlcNAc transferase governs flagellar motility through anti-repression. Genes Dev 2006, 20(23):3283-3295. 
64. Schirm M, Kalmokoff M, Aubry A, Thibault P, Sandoz M, Logan SM: Flagellin from Listeria monocytogenes is glycosylated with beta-O-linked Nacetylglucosamine. J Bacteriol 2004, 186(20):6721-6727.

65. Che FS, Nakajima Y, Tanaka N, Iwano M, Yoshida T, Takayama S, Kadota I, Isogai A: Flagellin from an incompatible strain of Pseudomonas avenae induces a resistance response in cultured rice cells. J Biol Chem 2000, 275(41):32347-32356.

66. Moens S, Michiels K, Vanderleyden J: Glycosylation of the flagellin of the polar flagellum of Azospirillum brasilense, a Gram-negative nitrogenfixing bacterium. Microbiology 1995, 141(10):2651-2657.

67. Guerry P, Ewing CP, Schirm M, Lorenzo M, Kelly J, Pattarini D, Majam G, Thibault $\mathrm{P}$, Logan $\mathrm{S}$ : Changes in flagellin glycosylation affect Campylobacter autoagglutination and virulence. Mol Microbiol 2006, 60(2):299-311.

68. Logan SM: Flagellar glycosylation - a new component of the motility repertoire? Microbiology 2006, 152(Pt 5):1249-1262.

69. Simon R, Priefer U, Pühler A: A broad host range mobilization system for in vivo genetic engineering: transposon mutagenesis in Gram-negative bacteria. Biotechnology 1983, 1:784-791.

70. Poole PS, Schofiel NA, Reid CJ, Drew EM, Walshaw DL: Identification of chromosomal genes located downstream of $d c t D$ that affect the requirement for calcium and the lipopolysaccharide layer of Rhizobium leguminosarum. Microbiology 1994, 140(10):2797-2809.

71. Priefer UB: Genes involved in lipopolysaccharide production and symbiosis are clustered on the chromosome of Rhizobium leguminosarum biovar viciae VF39. J Bacteriol 1989, 171(11):6161-6168.

72. Kovach ME, Elzer PH, Hill DS, Robertson GT, Farris MA, Roop RM, Peterson KM: Four new derivatives of the broad-host-range cloning vector pBBR1MCS, carrying different antibiotic-resistance cassettes. Gene 1995, 166(1):175-176.

doi:10.1186/1471-2180-10-219

Cite this article as: Tambalo et al:: Characterization and functional analysis of seven flagellin genes in Rhizobium leguminosarum bv. viciae. Characterization of $R$. leguminosarum flagellins. BMC Microbiology 2010 10:219.

\section{Submit your next manuscript to BioMed Central and take full advantage of:}

- Convenient online submission

- Thorough peer review

- No space constraints or color figure charges

- Immediate publication on acceptance

- Inclusion in PubMed, CAS, Scopus and Google Scholar

- Research which is freely available for redistribution 